\title{
In Silico Design of PCR Primers to Amplify the Salt Tolerance Gene in Soybean
}

\author{
Florentina Kusmiyati ${ }^{1}$, Sutarno ${ }^{2}$ and Bagus Herwibawa ${ }^{1, *}$, \\ ${ }^{1}$ Laboratory of Physiology and Crop Breeding, Faculty of Animal and Agricultural Sciences, Diponegoro University \\ Tembalang Campus, Semarang 50275, Central Java, Indonesia \\ ${ }^{2}$ Laboratory of Ecology and Crop Production, Faculty of Animal and Agricultural Sciences, Diponegoro University \\ Tembalang Campus, Semarang 50275, Central Java, Indonesia \\ *Corresponding author's email: bagus.herwibawa [AT] live.undip.ac.id
}

\begin{abstract}
Soybean yield is decreased because of many stresses such as salt stress. Ionic and osmotic stresses are the effects of salt stress. An effective way of maintaining sustainable production in salt-affected soil is through breeding high salt tolerance soybean, which can be detected by PCR. The optimal PCR plays an important role in gene expression analysis. The success of a PCR-based method largely depends on the optimal primer sequence analysis in silico prior to a wet-bench experiment. Here we described designing of primer using web-based tools. Many types of online primer design software are available, which can be used free of charge to design desirable primers. The objective was to design of PCR primers to amplify the salt tolerance gene in soybean. A highly conserved region of 411 bases was detected by Clustal Omega. Primers were predicted using Primer3 based on conserved region, considering ideal conditions for primer length, hairpin, dimer, Tm, and GC\%. The predicted forward and reverse primers were validated using NetPrimer. Both forward and reverse primers have shown significant similarity with salt tolerance gene and recommended to be used to amplify the salt tolerance gene in soybean.
\end{abstract}

Keywords - FASTA, Glycine max, NCBI, NetPrimer, Primer3

\section{INTRODUCTION}

Soil salinity is one of the major environmental factors contributing to the loss of crop productivity and quality [1]. Worldwide, more than 45 million hectares of irrigated agricultural land are affected by salinity, and then $50 \%$ of agricultural lands will be lost by the middle of the $21^{\text {st }}$ century [2]. It also become a severe threat to global food security and agricultural profits [3]. Soybean is classified as a salt-sensitive glycophyte, and salinity stress adversely inhibits all developmental stages [4], since it is the most important food crop because its content presents 40-42\% high quality protein, and 18-22\% oil comprising up of $85 \%$ unsaturated fatty acid [5]. However, salinity at higher levels generates both osmotic and ionic stess on soybean: an osmotic stress caused by reduced water availability in soil and an ionic stress due to imbalance of solutes in the cytosol [6]. Salt stress significantly affects on almost all development stages during the soybean life-cycle, including seed germination and seedling establishment [7]. Salt stress also significantly reduced plant height and total biomass [8], decrease the number of internodes and pods [9], decreases protein content and seed quality [10], and causes chlorosis and necrosis symptoms, in addition to general loss in productivity [11]. Meanwhile, the soybean yield decrease of up to $14-35.3 \%$ under salt stress [12].

Development of salt tolerant cultivars will help reduce loss of yield in soybean production under saline conditions [13]. A better understanding of the tolerance mechanisms and identifying the genes involved will enable breeders to develop new strategies to enhance salt tolerance of soybeans [6]. Genotypic variation in salt tolerance of soybean indicates there is potential for identifying genes that increase soybean production, and that the tolerant genotypes exhibit greater nodulation and nitrogen uptake [14]. The causal genes underlying the salt-tolerance locus have been identified by map-based cloning, and found that the dominant gene associated with salt tolerance (e.g. GmCHX1, GmSALT3, and GmST1) [15 - 20]. To speed up the process of creating a new generation with promising agronomic traits (salt tolerance elite soybean lines), salt related genes should be used in research via comparative genomics for plant breeding, transcriptome analysis for plant breeding, genotyping and marker-assisted breeding, and recombinant DNA technology [21]. Molecular markers in breeding programs have played an important role to be efficient in plant breeding with the aid of polymerase chain reaction (PCR) [22]. The use of this technique is becoming a common tool for detecting and quantifying expression profiles of selected genes [23].

PCR is an inexpensive technology for copying a specific sequence of DNA [24]. However the outcome of PCR can be 
influenced by many other conditions, such as the template DNA preparation and reaction conditions, designing a good pair of primer is a critical factor [25]. It is sensitive to mismatches between primer and template, and mismatches can lead to a distortion of the true relative abundance of targeted regions [26]. The primer which are unique for the target sequence to be amplified, so primer length, GC\%, melting and annealing temperature, 3' end specifity and 5' end stability are important features playing important role in the efficiency of a primer [27]. Primer design is essential to the successful outcome of a PCR experiment, and if the sets of primers are poorly designed, resulting in no production of copies or the occurrence of mismatches [28]. There are numerous web-based primer design software are available. Though most of the software are freely available, but use of these online software requires practice, as online manuals may not be available to assist users. Designing primers for salt tolerance genes through in silico studies can aid in the amplification of the genes that is a prerequisite for further studies. The objective was to design of PCR primers to amplify the salt tolerance gene in soybean.

\section{MATERIALS AND METHODS}

Nucleotide sequences were searched at the National Center for Biotechnology Information (NCBI, http:// ncbi.nlm.nih.gov/) through Entrez for GmCHX1, GmSALT3, and GmST1. The information about those sequences were reported in Table 1, and those sequences were subjected to Multiple Sequence Alignment (MSA) using Clustal Omega (EMBL-EBI, https://www.ebi.ac.uk/Tools/msa/clustalo/). Therefore the consensus sequence was further used as input for designing primer using Primer3 [29] (http://bioinfo.ut.ee/primer3-0.4.0/primer3/). The parameters were adjusted as follows: Pick left primer: check; Pick right primer: check; Sequence Id: GmST; Targets: skip this option; Excluded regions: blank; Product size ranges: 80-150 100-200; Number to return: 10; Max 3' Stability: 9; Max repeat mispriming: 12; Pair max repeat mispriming: 24; Max template mispriming: 12; Pair max template mispriming: 24; Primer size: min: 18, opt: 21, max: 24; Primer $\mathrm{T}_{\mathrm{m}}$ : min: 52, opt: 54, $\max$ : 58; Maximum $\mathrm{T}_{\mathrm{m}}$ difference: 2; Table of thermodynamic parameters: SantaLucia1998; Product $\mathrm{T}_{\mathrm{m}}$ : opt:50; Primer GC: $\min$ 35, opt: 65, max = 80; Max self complimentary: 4; Max 3' selfcomplimentary: 3; Max \#N: 0; Max poly-x: 3; Inside target penalty and outside target penalty: default; First base index: 1; GC clamp: 0; Conc. of monovalent cations: 50; Salt correction formula: SantaLucia1998; Conc. of divalent cations: 3.5; Conc. of dNTPs: 0.20; Annealing oligo concentration: 50; Objective fuction penalty weights for primers: $\mathrm{T}_{\mathrm{m}} \mathrm{Lt}$ : $1 \mathrm{Gt}: 1$, Size Lt:1 Gt:1, Self complementarity: 3, 3' self complementarity: 3; \#N's: 2; All other values: 0; Objective function penalty weight for primer pairs: Product $\mathrm{T}_{\mathrm{m}} \mathrm{Lt}: 1 \mathrm{Gt}: 1 \mathrm{~T}_{\mathrm{m}}$ difference: 2, Any complementarity: 3, 3' complementarity: 3, Primer penalty weight: 1 , All other values: 0 .

Table 1: Information regarding four nucleotide sequences belonging to Glycine max gene associated with salt tolerance

\begin{tabular}{lccc}
\hline \multicolumn{1}{c}{ Accession name } & Accession number & Sequence type & Sequence length \\
\hline GmCHX1 & KF879912 & Complete cds & 1131 \\
GmSALT3 & NM_001353420 & mRNA & 3259 \\
GmST1 & KU871394 & Partial cds & 411 \\
\hline
\end{tabular}

The primers obtained may or may not be optimal for PCR. It is important to analyze the primer melting temperature $\left(\mathrm{T}_{\mathrm{m}}\right)$, primer properties, primer secondary structures, primer rating, comprehensive reports, primer pairs, and comprehensive help using an additional software like NetPrimer (PREMIER Biosoft, https://www.premierbiosoft.com/netprimer/). Once the primers have been checked for these various features, it is important to also verify that the amplicon does not form secondary structures. This can be done using UNAFold (IDT, http://sg.idtdna.com/UNAFold). The parameters were adjusted as follows: Nucleotide type: DNA; Temperature: $60{ }^{\circ} \mathrm{C}$, Na concentration: $50 \mathrm{mM}$; Mg concentration: $3 \mathrm{mM}$; Suboptimality: 50 \%, Sequence type: linear; Max foldings: 20; Start position: blank; Stop position: blank. Again those nucleotide sequences were subjected for designing forward and reverse primer using GeneFisher2 (BiBiServ, http://bibiserv.cebitec.uni-bielefeld.de/genefisher2) followed by selection of suitable primer using NetPrimer. The parameters for primer prediction were adjusted as per previous case mentioned above. Furthermore, most gene expression assays contain cDNA made from total RNA collected from tissue, it is necessary to verify that the primers do not hybridize with another gene. So, the specifity of both forward and reverse primers was checked using BLAST (http://blast.ncbi.nlm.nih.gov/Blast.cgi).

\section{RESULTS AND DISCUSSION}

GmCHX1, GmSALT3, and GmST1 nucleotide sequences were taken for designing primer for salt tolerance gene in soybean. A highly conserved region (100\% identical) having 411 bases shown in Figure 1. The Primer3 predicted five forward and seven reverse primer based on consensus sequence (Table 2). Forward primers (F1-F5) having length 18 -20 bp, $40-50 \%$ GC content, 52- $54{ }^{\circ} \mathrm{C} \mathrm{Tm}$ and absence of secondary structure were finalized. F3 was the first candidate forward primer which should be chosen because it has $50 \% \mathrm{GC}$ content (recommendation $50-60 \%$ ), $53.80{ }^{\circ} \mathrm{C} \mathrm{Tm}$ (recommendation $52-58{ }^{\circ} \mathrm{C}$ ), zero for hairpin and dimer. Besides, F1 was the second candidate forward primer because it has $50 \% \mathrm{GC}$ content recommendation $50-60 \%$ ), $53.73{ }^{\circ} \mathrm{C} \mathrm{Tm}$ (recommendation $52-58{ }^{\circ} \mathrm{C}$ ), and zero for hairpin. In this case, there were 24492 primer pairs considered, 19501 were unacceptable product size, and 2132 had too large Tm difference. This information is useful in determining which parameters have been set too stiff. However there was no reverse primers (R1-R7) which have $50 \%$ GC content, so we supposed the primer obtained may not be optimal for PCR. 
In short, the key to developing good primers is to find a primers pair that are very specific, do not produce primer dimers, produce short amplicons, and are efficient enough to produce results that are consistent and reproducible [30]. According to [31], the predicted primers should be subjected to check various properties such as cross dimers are formed between forward and reverse primers, self dimers form between two primers of the same tipe, and hairpins form when primers fold back onto each other, to get the best primer pairs for PCR.

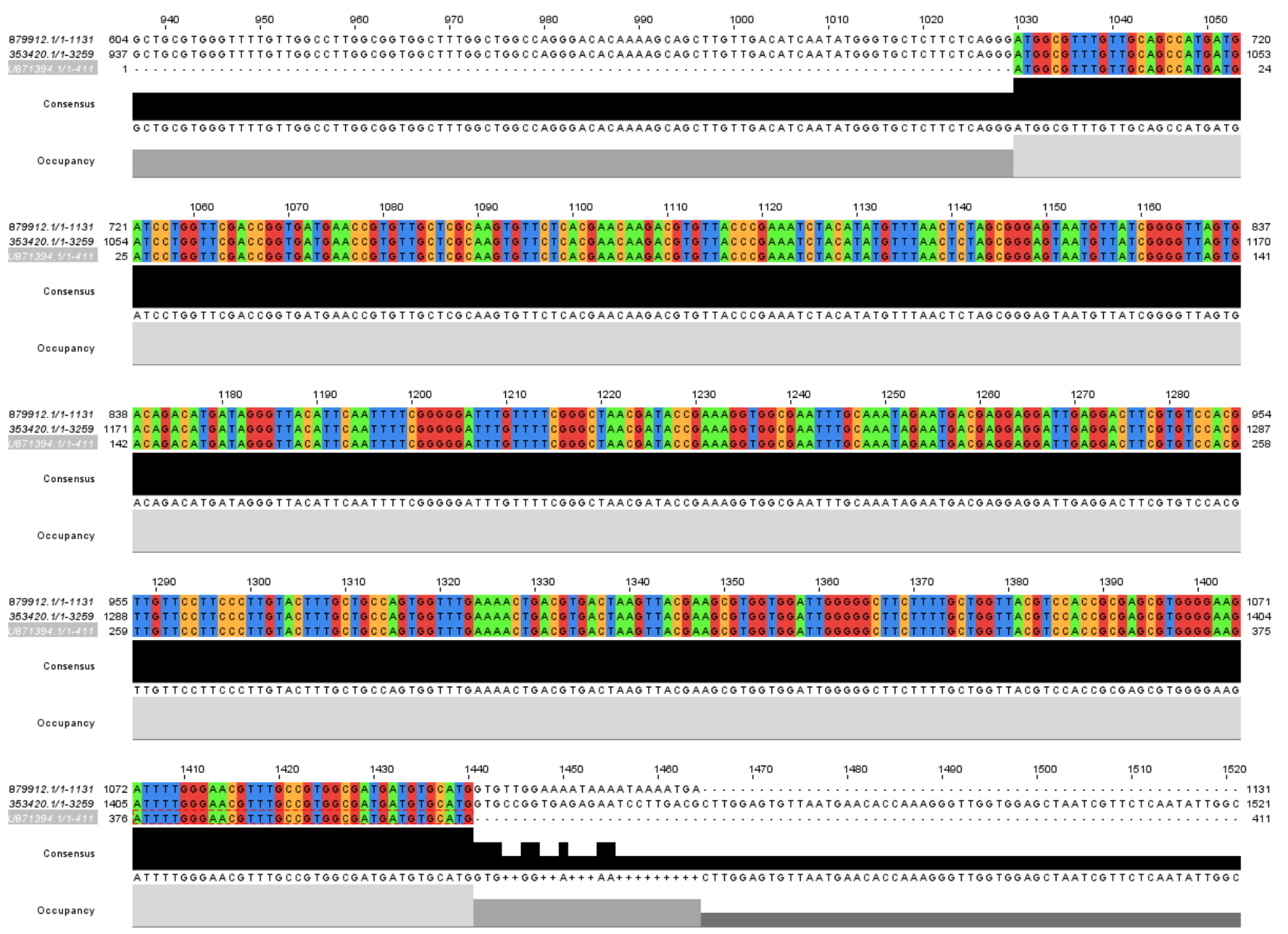

Figure 1: Jalview of ClustalW showing consensus sequence of highly conserved domain having 411 bases

Table 2: Various properties of forward and reverse primers

\begin{tabular}{|c|c|c|c|c|c|c|}
\hline Primer & Sequence (5'-3') & Length & Hairpin & Dimer & $T m$ & $\% G C$ \\
\hline F1 & GTGACAGACATGATAGGG & 18 & 0 & 1 & 53.73 & 50.00 \\
\hline $\mathrm{F} 2$ & TAACGATACCGAAAGGTG & 18 & 0 & 2 & 53.71 & 44.44 \\
\hline F3 & GGGAGTAATGTTATCGGG & 18 & 0 & 0 & 53.80 & 50.00 \\
\hline F4 & TAGTGACAGACATGATAGG & 19 & 0 & 1 & 52.76 & 42.11 \\
\hline F5 & TTAGTGACAGACATGATAGG & 20 & 0 & 0 & 53.86 & 40.00 \\
\hline$\overline{\mathrm{R} 1}$ & TACAAGGGAAGGAACAAC & 18 & $\overline{0}$ & 1 & $\overline{53.63}$ & $\overline{44.44}$ \\
\hline $\mathrm{R} 2$ & CAAAGTACAAGGGAAGGAA & 19 & 0 & 0 & 54.43 & 42.11 \\
\hline R3 & AGCAAAGTACAAGGGAAG & 18 & 0 & 0 & 54.06 & 44.44 \\
\hline R4 & CCTCCTCGTCATTCTATTT & 19 & 0 & 0 & 53.78 & 42.11 \\
\hline R5 & GTACAAGGGAAGGAACAA & 18 & 0 & 0 & 53.63 & 44.44 \\
\hline R6 & AGTACAAGGGAAGGAACA & 18 & 0 & 0 & 54.49 & 44.44 \\
\hline R7 & CAGCAAAGTACAAGGGAA & 18 & 0 & 0 & 54.41 & 44.44 \\
\hline
\end{tabular}

Note: Primer analized by Primer3. F: forward primer; R: reverse primer; Start: the position of the 5'base of the primer, for the forward primers it is the position of the leftmost base and for reverse primers it is the right most base; Length: length of the primer; Hairpin: form when primers fold back onto each other; Dimer: form between two primers of the same tipe; Tm: melting temperature of the primer; \% GC: the percent of G and C bases in the primer; ANY: self-complementary score of the primer; SELF: 3'selfcomplementary of the primer. 
Table 3: Various properties of F3 and F1 with reverse primers

\begin{tabular}{|c|c|c|c|c|c|c|}
\hline Forward & Sequence (5'-3') & Reverse & Sequence (5'-3') & $\begin{array}{l}\text { Cross } \\
\text { Dimer }\end{array}$ & $\begin{array}{c}\text { Self } \\
\text { Dimer }\end{array}$ & Hairpin \\
\hline \multirow[t]{7}{*}{ F3 } & GGGAGTAATGTTATCGGG & R1 & TACAAGGGAAGGAACAAC & -6.24 & 0 & 0 \\
\hline & & $\mathrm{R} 2$ & CAAAGTACAAGGGAAGGAA & 0 & -3.65 & 0 \\
\hline & & R3 & AGCAAAGTACAAGGGAAG & 0 & -3.65 & 0 \\
\hline & & $\mathrm{R} 4$ & СCTCCTCGTCATTCTATTT & -6.24 & 0 & 0 \\
\hline & & R5 & GTACAAGGGAAGGAACAA & -6.24 & -3.65 & 0 \\
\hline & & R6 & AGTACAAGGGAAGGAACA & -6.24 & -3.65 & 0 \\
\hline & & R7 & CAGCAAAGTACAAGGGAA & 0 & -3.65 & 0 \\
\hline \multirow[t]{7}{*}{$\overline{F 1}$} & GTGACAGACATGATAGGG & $\overline{\mathrm{R} 1}$ & TACAAGGGAAGGAACAAC & 0 & 0 & 0 \\
\hline & & $\mathrm{R} 2$ & CAAAGTACAAGGGAAGGAA & 0 & -3.65 & 0 \\
\hline & & R3 & AGCAAAGTACAAGGGAAG & 0 & -3.65 & 0 \\
\hline & & $\mathrm{R} 4$ & СCTCCTCGTCATTCTATTT & -5.67 & 0 & 0 \\
\hline & & R5 & GTACAAGGGAAGGAACAA & 0 & -3.65 & 0 \\
\hline & & R6 & AGTACAAGGGAAGGAACA & 0 & -3.65 & 0 \\
\hline & & R7 & CAGCAAAGTACAAGGGAA & 0 & -3.65 & 0 \\
\hline
\end{tabular}

Note: Primer analized by NetPrimer. F: forward primer; R: reverse primer; Start: the position of the 5 'base of the primer, for the forward primers it is the position of the leftmost base and for reverse primers it is the rightmost base; Cross Dimer: form between forward and refers primers; Hairpin: form when primers fold back onto each other; Dimer: form between two primers of the same tipe; Tm: melting temperature of the primer

Table 4: Secondary structure analysis

\begin{tabular}{cccccc}
\hline Primer & Sequence (5'-3') & $\Delta \boldsymbol{G}$ & $\boldsymbol{T}_{\boldsymbol{m}}$ & $\Delta \boldsymbol{H}$ & $\Delta \boldsymbol{S}$ \\
\hline F1 & GTGACAGACATGATAGGG & -0.52 & 33 & -19.6 & -64.01 \\
R1 & TACAAGGGAAGGAACAAC & - & - & - & -
\end{tabular}

Note: Primer analized by UNAFold. F: forward primer; R: reverse primer; Start: the position of the 5'base of the primer, for the forward primers it is the position of the leftmost base and for reverse primers it is the rightmost base
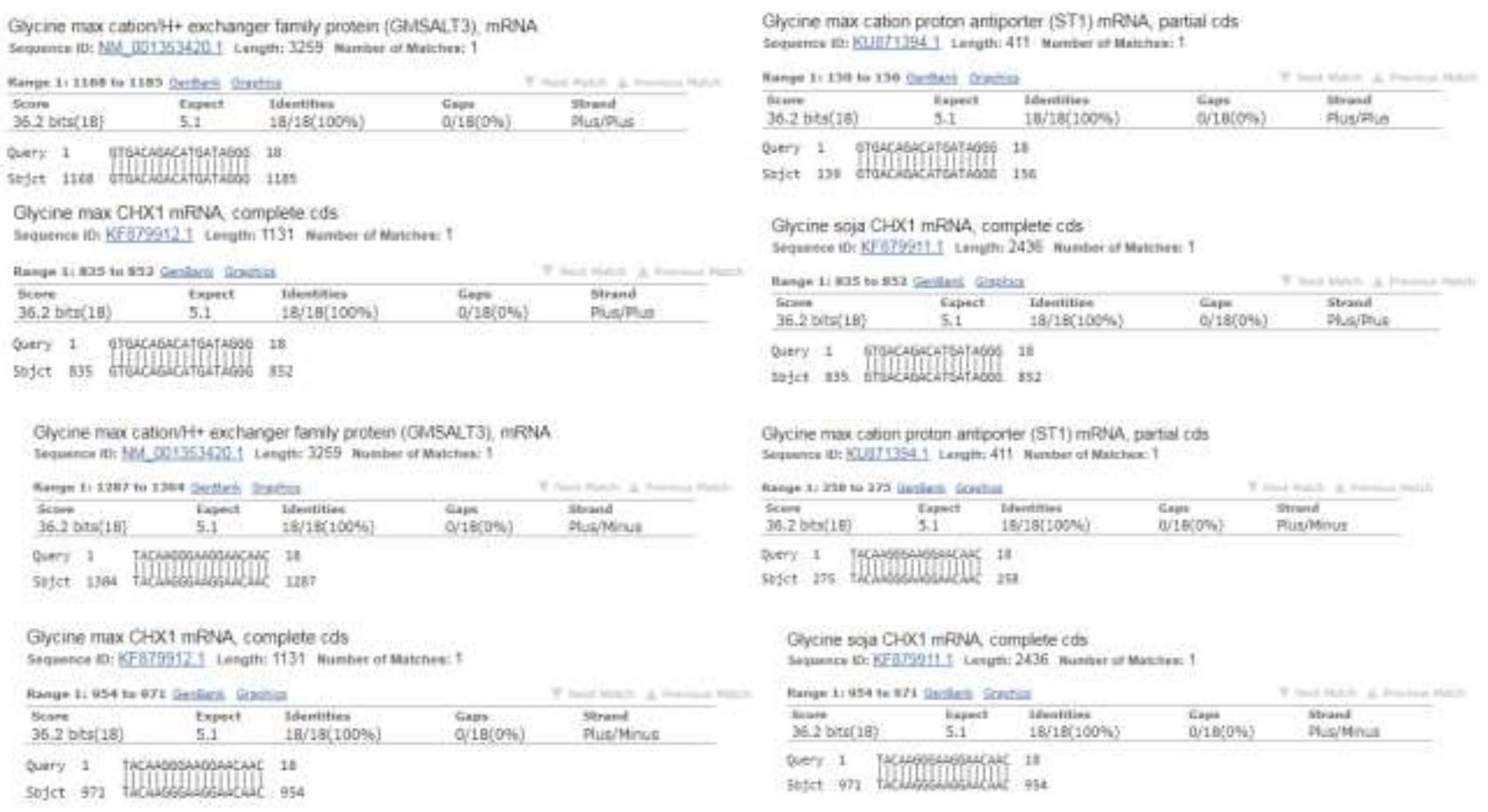

Figure 2: BLAST result for the designed forward and reverse primers

The primer properties were checked between F3 and F1 with reverse primers by NetPrimer (Table 3). F3 and reverse primer pairs showed cross dimer and self dimer together were found in R5 and R6, so this pairs should be avoided because it showed false positives. It is, therefore, critical to design primers that reduce the chance of dimerization and non-specific amplification. However, F1 and R1 primer pair showed zero for cross dimer, self dimer, and hairpin. Table 4 explain that 
R1 was not formed a structure and F1 only have a structure. The primer specifity was checked for F1 and R1 primers (Figure 2) which has shown significant alignment with salt tolerance gene. Pridicted primers are important steps before primer synthesis to be used for PCR. In the current study a systematic approach has been made to predict a theoretical primers. The predicted forward and reverse primers have shown significant similarity with for salt tolerance gene in soybean. Hence it may be considered that the designed primers would be used for amplification from heterologous sources leading to discovery of new genes and allele mining.

\section{CONCLUSIONS}

A highly conserved region of 411 bases was detected by Clustal Omega. Primers were predicted using Primer3 based on conserved region, considering ideal conditions for primer length, hairpin, dimer, Tm, and GC\%. The predicted forward and reverse primers were validated using NetPrimer. Both forward (GTGACAGACATGATAGGG) and reverse (TACAAGGGAAGGAACAAC) primers have shown significant similarity with salt tolerance gene and recommended to be used to amplify the salt tolerance gene in soybean.

\section{ACKNOWLEDGEMENT}

We want to convey our gratitude to The Directorate General of Research and Community Service, The Ministry of Research, Technology and Higher Education of The Republic of Indonesia for the research grant through scheme of “ Superior Research of University", and Diponegoro University for the continuous support, particularly to the Institute of Research and Community Services.

\section{REFERENCES}

[1] R. M. A. Machado, R. P. Serralheiro, "Soil Salinity: Effect on Vegetable Crop Growth. Management Practices to Prevent and Mitigate Soil Salinization", Horticulturae, vol. 3, no. 2: 30, 2017. doi: 10.3390/horticulturae3020030

[2] M. Hasanuzzaman, K. Nahar, Md. M. Alam, P.C. Bhowmik, Md. A. Hossain, M. M. Rahman, M. N. V. Prasad, M. Ozturk, M. Fujita, "Potential Use of Halophytes to Remediate Saline Soils", BioMed Research International, Article ID 589341, 2014. doi:10.1155/2014/589341

[3] K. Butcher, A. F. Wick, T. DeSutter, A. Chatterjee, J. Harmon, "Soil Salinity: A Threat to Global Food Security", Agronomy Journal, vol. 108, no. 6, pp. 2189-2200, 2016. doi: 10.2134/agronj2016.06.0368

[4] R. Guan, J. Chen, J. Jiang, G. Liu, Y. Liu, L. Tian, L. Yu, R. Chang, L. Qiu, "Mapping and validation of a dominant salt tolerance gene in the cultivated soybean (Glycine max) variety Tiefeng 8", The Crop Journal, vol. 2, no. 6, pp. 358-365, 2014. doi: 10.1016/j.cj.2014.09.001

[5] F. Anwar, G. M. Kamal, F. Nadeem, G. Shabir, "Variations of Quality Characteristics Among Oils of Different Soybean Varieties", Journal of King Saud University - Science, vol. 28, no. 4, pp. 332-338, 2016. doi: 10.1016/j.jksus.2015.10.001

[6] S. Ren, C. Lyle, G. Jiang, A. Penumala, "Soybean Salt Tolerance 1 (GmST1) Reduces ROS Production, Enhances ABA Sensitivity, and Abiotic Stress Tolerance in Arabidopsis thaliana", Frontiers in Plant Science, vol. 7, Article 445, 2016. doi: 10.3389/fpls.2016.00445

[7] K. Shu, Y. Qi, F. Chen, Y. Meng, X. Luo, H. Shuai, W. Zhou, J. Ding, J. Du, J. Liu, F. Yang, Q. Wang, W. Liu, T. Yong, X. Wang, Y. Feng, W. Yang, "Salt Stress Represses Soybean Seed Germination by Negatively Regulating GA Biosynthesis While Positively Mediating ABA Biosynthesis”, Frontiers in Plant Science, vol. 8, Article 1372, 2017. doi: 10.3389/fpls.2017.01372

[8] C. Bustingorri, R. S. Lavado, "Soybean Growth under Stable Versus Peak Salinity", Scientia Agricola, vol. 68, no. 1, pp. 102-108, 2011. doi: 10.1590/S0103-90162011000100015

[9] A. E. Sabagh, M. S. Islam, A. Ueda, H. Saneoka, C. Barutcular, "Increasing Reproductive Stage Tolerance to Salinity Stress in Soybean”, International Journal of Agriculture and Crop Sciences, vol. 8, no. 5, pp. 738-745, 2015.

[10] J. Zhang, D. Yang, M. Li, L. Shi, "Metabolic Profils Reveal Changes in Wild and Cultivated Soybean Seedling Leaves under Salt Stress”, PloS ONE, vol. 11, no. 7: e0159622, 2016. doi: 10.1371/journal.pone.0159622

[11] F. Ledesma, C. Lopez, D. Ortiz, P. Chen, K. L. Korth, T. Ishibashi, A. Zeng, M. Orazaly, L. Florez-Palacios, “A Simple Greenhouse Method for Screening Salt Tolerance in Soybean”, Crop Science, vol. 56, no. 2, pp. 585-594, 2016. doi: 10.2135/cropsci2015.07.0429

[12] Y. He, Y. Chen, C. L. Yu, K. X. Lu, Q. S. Jiang, J. L. Fu, G. M. Wang, D. A. Jiang, "Photosynthesis and Yield Traits in Different Soybean Lines in Response to Salt Stress", Photosynthetica, vol. 54, no. 4, pp. 630-635, 2016. doi: 10.1007/s11099-016-0217-7 
[13] J. D. Lee, J. G. Shannon, T. D. Vuong, H. T. Nguyen, "Inheritance of Salt Tolerance in Wild Soybean (Glycine soja Sieb. And Zucc.) Accession PI483463”, Journal of Heredity, vol. 100, no. 6, pp. 798-801, 2009. doi: 10.1093/jhered/esp027

[14] Y. Song, T. Nakajima, D. Xu, K. Homma, M. Kokubun, "Genotypic Variation in Salinity Tolerance and Its Association with Nodulation and Nitrogen Uptake in Soybean”, Plant Production Science, vol. 20, no. 4, pp. 490498, 2017. doi: 10.1080/1343943X.2017.1360140

[15] H. Chen, H. He, D. Yu, "Overexpression of A Novel Soybean Gene Modulating $\mathrm{Na}^{+}$And K ${ }^{+}$Transport Enhances Salt Tolerance in Transgenic Tobacco Plants”, Physiologia Plantarum, vol. 141, no. 1, pp. 11-18, 2011. doi: 10.1111/j.1399-3054.2010.01412.X

[16] R. Guan, Y. Qu, Y. Guo, L. Yu, Y. Liu, J. Jiang, J. Chen, Y. Ren, G. Liu, L. Tian, L. Jin, Z. Liu, H. Hong, R. Chang, M. Gilliham, L. Qiu, "Salinity Tolerance in Soybean is Modulated by Natural Variation in GmSALT3", The Plant Journal, vol. 80, no.6, pp. 937-950, 2014. doi: 10.1111/tpj.12695

[17] WYF. Li, F.L. Wong, S.N. Tsai, T.H. Phang, G.H. Shao, H.M. Lam, "Tonoplast-Located Gmclc1 And GmNHX1 from Soybean Enhance NaCl Tolerance in Transgenic Bright Yellow (BY)-2 Cells”, Plant, Cell and Environment, vol. 29, no. 6, pp. 1122-1137, 2006. doi: 10.1111/j.1365-3040.2005.01487.x

[18] G.Z. Luo, H.W. Wang, J. Huang, A.G. Tian, Y.J. Wang, J.S. Zhang, S.Y. Chen, “A Putative Plasma Membrane Cation/Proton Antiporter from Soybean Confers Salt Tolerance in Arabidopsis”, Plant Molecular Biology, vol. 59, no. 5, pp. 809-820, 2005. doi: 10.1007/s11103-005-1386-0

[19] S. Ren, C. Lyle, G. Jiang, A. Penumala, "Soybean Salt Tolerance 1 (GmST1) Reduces ROS Production, Enhances ABA Sensitivity, and Abiotic Stress Tolerance in Arabidopsis thaliana", Frontiers in Plant Science, vol. 7, no. 445, 2016. doi: 10.3389/fpls.2016.00445

[20] X. Qi, M.W. Li, M. Xie, X. Liu, M. Ni, G. Shao, C. Song, A.K.Y. Yim, Y. Tao, F.L. Wong, S. Isobe, C.F. Wong, K.S. Wong, C. Xu, C. Li, Y. Wang, R. Guan, F. Sun, G. Fan, Z. Xiao, F. Zhou, T.H. Phang, X. Liu, S.W. Tong, T.F. Chan, S.M.Yiu, S. Tabata, J. Wang, X. Xu, H. M. Lam, "Identification of A Novel Salt Tolerance Gene in Wild Soybean by Whole-Genome Sequencing", Nature Communications, vol. 5, Article 4340, 2014. doi: $10.1038 /$ ncomms5340

[21] W. Wang, X. H. Cao, M. Miclaus, J. Xu, W. Xiong, "The Promise of Agriculture Genomics”, International Journal of Genomics, Article ID 9743749, 2017. doi: 10.1155/2017/9743749

[22] M.F. Parera, J. Racedo, M.G. Garcia, E.M. Pardo, C.M.L. Rocha, I.G. Orce, M.A. Chiesa, M.P. Filippone, B. Welin, A.P. Castagnaro, "Use of Molecular Markers to Improve the Agro-Industrial Productivity in the North West of Argentina", Molecular Biology, vol. 5, no. 1, Article 153, 2016. doi: 10.4172/2168-9547.1000153

[23] S.A. Deepak, K.R. Kottapalli, R. Rakwal, G. Oros, K.S. Rangappa, H. Iwahashi, Y. Masuo, G.K. Agrawal, "RealTime PCR: Revolutionizing Detection and Expression Analysis of Genes”, Current Genomics, vol. 8, no. 4, pp. 234251, 2007. doi: 10.2174/138920207781386960

[24] M. C. F. Thomsen, H. Hasman, H. Westh, H. Kaya, O. Lund, "RUCS: Rapid Identification of PCR Primers for Unique Core Sequences", Bioinformatics, vol. 33, no. 24, pp. 3917-3921, 2017. doi: 10.1093/bioinformatics/btx526

[25] J. Ye, G. Coulouris, I. Zaretskaya, I. Cutcutache, S. Rozen, T.L. Madden, "Primer-BLAST: A Tool to Design TargetSpecific Primers for Polymerase Chain Reaction”, BMC Bioinformatics, vol. 13, no. 134, 2012. doi: 10.1186/14712105-13-134

[26] S. J. Green, R. Venkatramanan, A. Naqib, "Deconstructing the Polymerase Chain Reaction: Understanding and Correcting Bias Associated with Primer Degeneracies and Primer-Template Mismatches”, PloS ONE, vol. 10, no. 5: e0128122, 2015. doi: 10.1371/journal.pone.0128122

[27] M. A. Nadeem, M. A. Nawaz, M. Q. Shahid, Y. Dogan, G. Comertpay, M. Yildiz, R. Hatipoglu, F. Ahmad, A. Alsaleh, N. Labhane, H. Ozkan, G. Chung, F. S. Baloch, "DNA Molecular Markers in Plant Breeding: Current Status and Recent Advancements in Genomic Selection and Genome Editing", Biotechnology and Biotechnological Equipment, 2017. doi: 10.1080/13102818.2017.1400401

[28] Analytical Methods Committee, AMCTB No. 59, "PCR- The Polymerase Chain Reaction", Analytical Methods, vol. 6, no. 2, pp. 333-226, 2014. doi: 10.1039/C3AY90101G

[29] S. Rozen, and H. Skaletsky, Primer3 on the WWW for General Users and for Biologist Programmers, Bioinformatics Methods and Protocols (Humana Press Inc.), USA, 2000

[30] P. S. Gangasagar, S. Rath, and I. C. Mohanty, "In Silico Design of Primer for In Vitro Cloning of DREB1A: A Regulatory Gene Associated with Abiotic Stress", Biotechnology, Bioinformatics and Bioengineering, vol. 2, no. 1, pp. 603-609, 2012

[31] B. Thornton, and C. Basu, "Real-Time PCR (qPCR) Primer Design Using Gree Online Software", Biochemistry and Molecular Biology Education, vol. 39, no. 2, pp. 145-154, 2011. doi: 10.1002/bmb.20461 